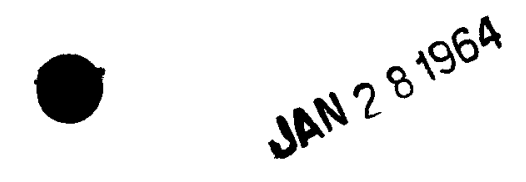

A

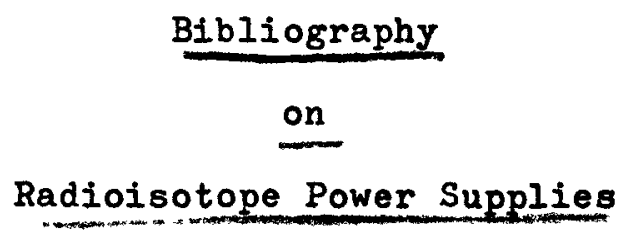

August, 1963

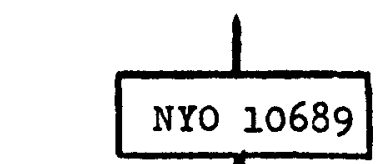

Facsimile Price $\$$

Microfilm Price $\$$

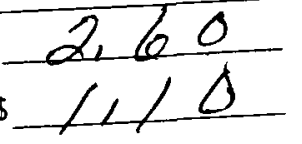

Available from the

Office of Technical Services

Department of Commerce

Washington 25, D. C.

$$
\begin{gathered}
\text { Daniel M. Axelrod } \\
\text { \& } \\
\text { Joseph P. Novarro } \\
\text { Reactor Development Division }
\end{gathered}
$$

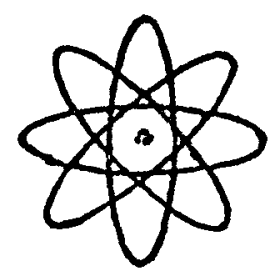

\title{
UNITED STATES ATOMIC ENERGY COMMISSION




\section{DISCLAIMER}

This report was prepared as an account of work sponsored by an agency of the United States Government. Neither the United States Government nor any agency Thereof, nor any of their employees, makes any warranty, express or implied, or assumes any legal liability or responsibility for the accuracy, completeness, or usefulness of any information, apparatus, product, or process disclosed, or represents that its use would not infringe privately owned rights. Reference herein to any specific commercial product, process, or service by trade name, trademark, manufacturer, or otherwise does not necessarily constitute or imply its endorsement, recommendation, or favoring by the United States Government or any agency thereof. The views and opinions of authors expressed herein do not necessarily state or reflect those of the United States Government or any agency thereof. 


\section{DISCLAIMER}

Portions of this document may be illegible in electronic image products. Images are produced from the best available original document. 
LEGAL NOTICE

This report was prepared as an account of Government sponsored work. Neither the United States, nor the Commission, nor any person acting on behalf of the Commission:

A. Makes any warranty or representation, expressed or implied, with respect to the accuracy, completeness, or usefulness of the information contained in this report, or that the use of any information, apparatus, method, or process disclosed in this report may not infringe privately owned rights; or

B. Assumes any liabilities with respect to the use of, or for damages resulting from the use of any information, apparatus, method, or process disclosed in this report.

As used in the above, "person acting on behalf of the Commission" includes any employee or contractor of the Commission, or employee of such contractor, to the extent that such employee or contractor of the Commission, or employee of such contractor prepares, disseminates, or provides access to, any information pursuant to his employment or contract with the Commission, or his employment with such contractor. 
NYO 10689

A

BIBLIOGRAPHY

ON

RADIOISOTOPE POWER SUPPLIES

AUGUST, 1963

DANIEL M. AXELROD

Project Engineer

and

JOSEPH NOVARRO

Nuclear Engineering Trainee

REACTOR DEVELOPMENT DIVISION

NEW YORK OPERATIONS OFFICE

U. S. ATOMIC ENERGI COMMISSION 


\section{FOREWORD}

This Bibliography on Radiolsotope Power Supplies (RIPS) was prepared as a supporting attachment to "Recommendations for a Program Development Plan for Radioisotope Power Supplies, FY 1964-FI 1968" (NYO 10609, August, 1963). The emphasis in this listing is on reports issued under AEC Division of Reactor Development RIPS systems development contracts. However, selected additional references of interest in the RIPS development program have also been included. Since timeliness was a more important consideration than completeness in issuing this Bibliography, it should not be considered a complete listing of all reports in this field.

In addition to its use by the Atomic Energy Commission and AEC RIPS contractors, the bibliography should be useful to potential RIPS users and firms interested in performing work for the Commission in this field. 
TABLE OF CONTENTS

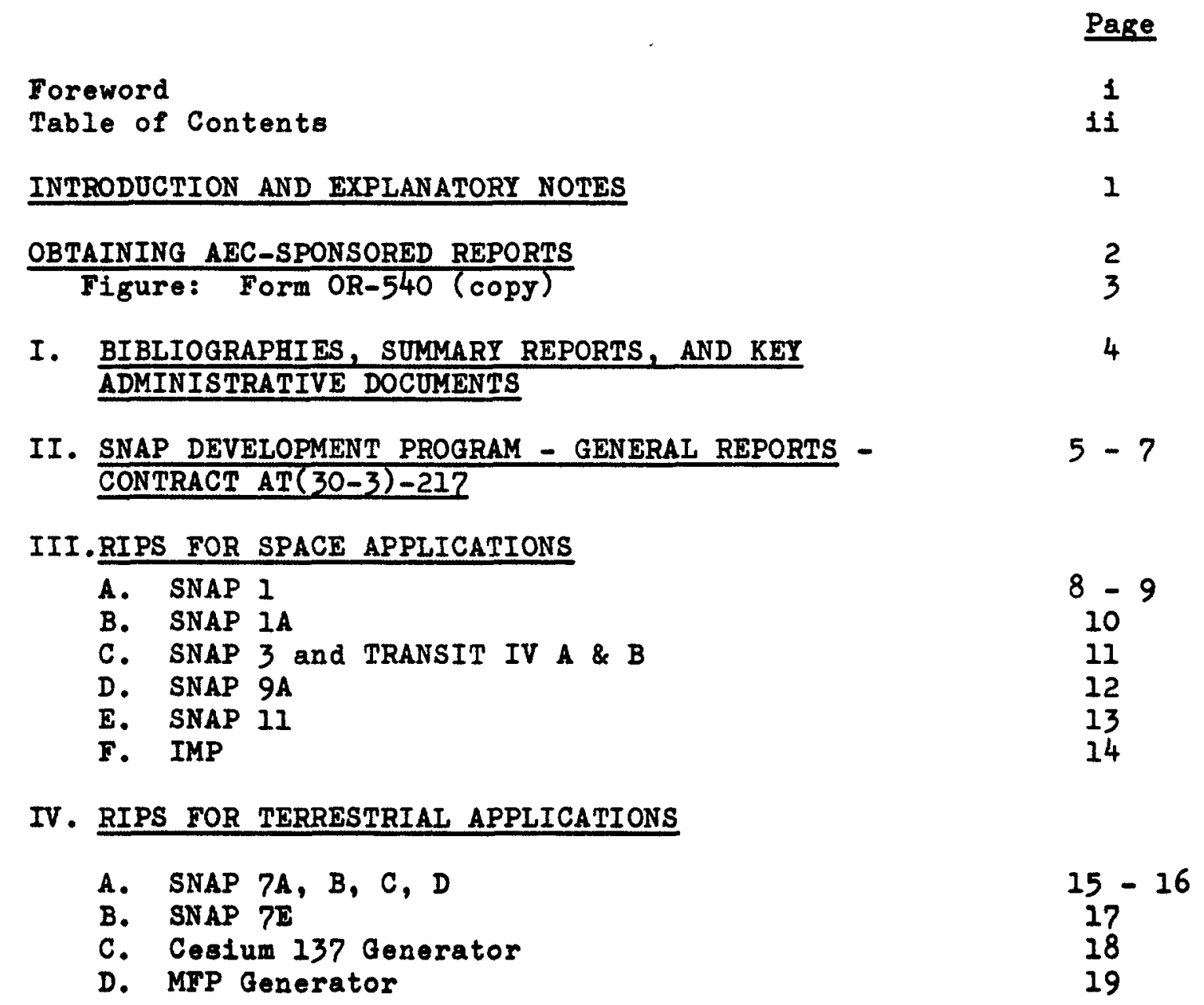

V. RIPS RESEARCH AND DEVELOPMENT

A. Thermionic R \& D

Advanced SNAP Technology - Contract AT(30-3)-217, 20

Task V and SNAP 13 Contract AT(30-1)-3060

Barium Vapor Filled Thermionic Development 21

B. Other Research \& Development Reports 22

APPENDIX I. Selected Additional References in $23-24$

Unclassified Iiterature

APPENDIX II. Unclassified Summary of Radioisotope 25 Power Supplies

APPENDIX III. Sample List of Specifications and $26-27$ Drawings for a Radioisotope Power Supply 


\section{INTRODUCTION AND EXPLANATORY NOTES}

This list represents a selected bibliography of AEC-sponsored reports pertaining to radioisotope power supplies, with emphasis on systems development reports.

Distribution

Because of security classification and the nature of the program, the distribution of these were categorized into the following:

Limited -- Atomic Energy Commission Offices (copies not available for distribution

Special -- (Government and industry installations as JC -33 c-92A (prescribed by the cognizant AEC Operations (office

(Obtain from DTIE, Oak Ridge)

\section{Classification}

JNCL - Onclassified

CDI - Confidential - Defense Information

CRD - Confidential - Restricted Data

SDI - Secret - Defense Information

SRD - Secret - Restricted Data

Author

MWD - Martin Company, Nuclear Division

TRW - Thompson-Ramo-Wooldridge Co.

GI - General Instrument Corp. 


\section{OBTAINING AEC-SPONSORED REPORTS}

\section{AEC Contractors}

AEC Contractors may obtain the reports they require for performance of their contract free of charge. Form OR-540, Official Report Request, (shown on the next page), should be completed to obtain any of the following AEC reports. Those reports which are determined to be applicable to the work of the contractor by the Division of Technical Information Extension (DTIE), Oak Ridge, will be trnasmitted. In the case of classified reports, a statement of the applicability of the report to the work being performed will expedite the review, particularly when new projects are initiated.

\section{Other Organizations}

Firms who do not hold AEC contracts but wish to obtain reports should contact DTIE, Oak Ridge. Access to classified reports will require appropriate clearances and establishing a need-to-know.

For furtherinformation consult the following references (available free from DTIE):

$$
\begin{aligned}
& \text { TID } 485 \text { - Technical Information Services of the USAEC - } \\
& \text { comprehensive descriptions of all the services } \\
& \text { available to the contractor at DTIE. } \\
& \text { NYO } 2834 \text { - Guide to AEC Report Preparation and Dissemination - } \\
& \text { fully describes report format requirements and } \\
& \text { services of DTIE. } \\
& \text { Availability of AEC Technical Information - Glossary of } \\
& \text { terms describing procedures for acquiring reports. }
\end{aligned}
$$




\section{OR 540 (copy)}

OFFICIAL REPORT REQUEST (Not to be used for reports required under an access permil)

Tor U. S. Aromic Energy Commission

Office of Tachnical Information Extension

P. O. Dox 62

Oak Ridoe, Tenn.

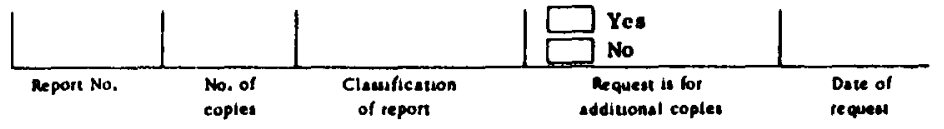

PLEASE NOTE; Use only for specificaliy identifted reports. Submit separate forms for each item requested. Submit in triplicale. Saple together the three form s for each request.

If report is classtifed, out side the categories which you a re authorized to recelve, please furnish justification with this request.

The Ofice of Technical information Extension can frequently supply unclasuified reports only on loan in full-sise copy or for retention in microcard form. In order to expedite the fllling of your request, please indicate your preference should OTI Extenslon etock of full-ake coples be dopleted. $\square$ Loan in fult-size copy $\square$ Microcard retention

If report number is not known or if report is not an AEC series, include

Send to: (enter complete malling address)

the information requested below.

Title

Author(s)

Originating organization

Date of report

Contract number of report

This report is requlred in connection with work tor the U. S. Government under contract No.

Requested for

Signed

FORM OR -54QREV 7-60)

(Front)

1. Report not In OTI Extension. We are attempting to obtain it.

2. Not yet avalable. You will receive the report automatically when distribution is made.

3. Report is available in

4. Cannot be Identified from the information furnished. Furnish additional information such as author, issuing organtzation, contract number, and source of reference.

5. No a vailable coples. Additlonal coples have been requested from Issuing office.

6. Copy has been furntshed previously on the date indicated.

- $\square$ Additional copies are not available.

$\square$ Are additional coples needed?

7. The report has not been 1 ssued to date. Per

8. See letter from OTI Extension dated

9. We have been informed by the issuing organization that this report recelved internal distribution only.

10. Report is $\square$ Jobsolete: $\square$ sdperseded by

11. Not an AEC publication. Suggested source is noted below.

12. Other 
I. BIBLIOGRAPHIES, SUMMARY REPORTS, AND KEY ADMINISTRATIVE DOCUNEITS

\section{Bibliographies}

1. B1bliography of SNAP Reports, MND-P-2413, Aug. 1960

Iists reports prepared under Contract AT(30-3)-217. (UncI)

Gany of the references listed have been included in this bibliography).

2. Sidney F. Lanier \& Henry D. Raleigh, Direct Energy Conversion and Systems for Nuclear Auxiliary Power (SNAP), A Literature Search TID-3561 (Rev. 3) January 1963 (Uncl.)

3. Smith, E. H. and W. Bowes, Isotopla Power Sources ... A Compendium: Property and Processes Review MND-P-2581

$$
\text { June } 1961 \text { (contract } \operatorname{AT}(30-3)-217 \text { ) }
$$

Volume I - List of References (Uncl)

Volume II - Abstracts of Key References (S-RD)

Volume III- Abstracts of Key References (UncI)

4. U. S. Naval Rsch. Lab, Direct Energy Conversion Literature Abstracts, Compiled in the library Branch, Tech. Info. Div., J. S. Naval Rach. Iab., Dec. 1962. (Avallable from O. T. 8.)

\section{Summary Reports}

1. J. G. Morse, "RIPS - Radioisotope-Fueled Power supplies: Lecture Presented at University of California," Los Angeles, California, July 1961, The Martin Co., Baltimore, Md. (Uncl)

2. Radioisotope'- Pueled Generator Compendium and Parametric Study. C. Fink \& T. Bustard, June 1963 MND-2994 (C-RD)

\section{Eer Administrative Documents}

1. Recommendations for a Program Development Plan

For Radiol sotope Power Bupplies, FI1964-FI1968, NY0-10609

Reactor Development Dirision, NYOpns Office, August, 1963

(Iinted Distribution) (C-RD)

2. Present and Potential Annual Arailability of Isotopic Power Fuels, Div. of Isotopes Development, April 1962

3. Classification Guide for the Isotopic Power Prosram CG-RIP-1 AEC Division of Classification, May 1962, C-DI 
II.

SNAP Development Prograns - General Reports-CorTRACT AT(30-) -217

\begin{tabular}{|c|c|c|c|c|c|c|}
\hline Report No. & Titlo & Date & $\begin{array}{l}\text { Distri- } \\
\text { bution }\end{array}$ & & Author & flcat \\
\hline MND -1001 & $\begin{array}{l}\text { Power from Radioisotopes } \\
\text { Survey Report }\end{array}$ & $10 / 56$ & Special & & F. Bittman & SRD \\
\hline MND-1002 & $\begin{array}{l}\text { Power fron. Radioisotopes } \\
\text { Conceptual Design Report }\end{array}$ & $1 / 57$ & Special & & K. Johnson & SRD \\
\hline MND-1138 & $\begin{array}{l}\text { Interin Hasards Inalysis } \\
\text { Report }\end{array}$ & $8 / 57$ & Special & . & s. Clark & SRD \\
\hline MND-1086 & $\begin{array}{l}\text { Radioisotope Fueled } \\
\text { Auxiliary Power Unit } \\
\text { Quarterly Progress Report }\end{array}$ & $\begin{array}{r}5 / 57 \\
\text { To. } 1\end{array}$ & $\mathbf{S}_{\text {pecial }}$ & & $\begin{array}{l}\text { C. Silverstein } \\
\text { R. Behmer }\end{array}$ & SRD \\
\hline MND-1123 & Report Ho. 2 & $8 / 57$ & Special & & K. Johnson & SRD \\
\hline MND-P-1175 & Report Ho. 3 & $10 / 57$ & $n$ & & K. Johnson & SRD \\
\hline MND-P-3001 & Report Ho. 4 & $2 / 58$ & " & & K. Johnson & SDI \\
\hline MRD-P-3002 & Report No. 5 & $5 / 58$ & $n$ & & K. Johnson & SDI \\
\hline MND-P-3003 & Report 10. 6 & $9 / 58$ & $\underline{n}$ & & K. Johnson & SDI \\
\hline MND-P-3004 & Report No. 7 & $11 / 58$ & " & & K. Johnson & SDI \\
\hline MND-P-3005 & Report Ha. 8 & $2 / 59$ & n & & K. Johnson & SDI \\
\hline MND-P-3006 & Report Ho. 9 & $6 / 59$ & n & & D. Harrey & SDI \\
\hline MND-P-3007 & Report Ho. 10 & $10 / 59$ & $\boldsymbol{n}$. & - & D. Harvej & SDI \\
\hline MND-P-3008 & Report No. 11 & $11 / 59$ & $n$ & & D. Harvey & SDI \\
\hline MND-P-3009 & $\begin{array}{l}\text { SHAp Prograhs QuarterlJ } \\
\text { Report Ho. } 12 \text { (Classi- } \\
\text { fied Section) }\end{array}$ & $3 / 59$ & Special & & D. Harvej & SDI \\
\hline
\end{tabular}


II.

SNAP Development Programs

- General Reports

- CONTRACT AT(30-3)-217 (Cont'd)

\begin{tabular}{|c|c|c|c|c|}
\hline Report No. & Title & $\begin{array}{l}\text { Distri- } \\
\text { bution }\end{array}$ & Author & ssific \\
\hline MND-P-3009-1 & $\begin{array}{l}\text { SNAP Programs Quarterly } \\
\text { Report No. I (Unclassi- } \\
\text { fied Section) }\end{array}$ & Special & J. Morse & UNCL \\
\hline MND-P- 3010 & $\begin{array}{l}\text { SNAP Programs Quarterly } 7 / \\
\text { Report No. } 2\end{array}$ & Special & D. Harvey & UNCL \\
\hline MND-P-3011 & $\begin{array}{l}\text { SNAP Programs Quarterly } 8 / 60 \\
\text { Report No. } 3\end{array}$ & Special & D. Harvey & UNCL \\
\hline MND-P-2047 & $\begin{array}{l}\text { Hazards Summary Report - } 6 / 59 \\
\text { Three-Watt Polonium-210 } \\
\text { Fueled Thermoelectric } \\
\text { Generator }\end{array}$ & Limited & W. Crane & SDI \\
\hline MND-P-2048 & $\begin{array}{l}\text { Hazards Summary Report } 6 / 59 \\
\text { for Two-Watt Strontium-90 } \\
\text { Fueled Thermoelectric } \\
\text { Generator }\end{array}$ & Limited & W. Crane & SDI \\
\hline MND-P-2049 & $\begin{array}{l}\text { Hazards Summary Report } 6 / 59 \\
\text { for Two-Watt Promethium-147 } \\
\text { Fueled Thermoelectric Generator }\end{array}$ & Limited & W. Crane & SDI \\
\hline MND-P-2148 & $\begin{array}{l}\text { Ten Watt Radioisotope } \quad 1 / 60 \\
\text { Thermoelectric Power Supply } \\
\text { for Project Transit Satellite }\end{array}$ & & D. J. Knight & UNCL \\
\hline MND-P-2333 & $\begin{array}{l}\text { Summary Report of Americium } 3 / 60 \\
\text { Process to be Performed by } \\
\text { Martin Company }\end{array}$ & Limited & G. Dix & UNCL \\
\hline MND-P-2347 & $\begin{array}{l}\text { Final Hazards Summary } 5 / 60 \\
\text { Report of Americium Process } \\
\text { to be Performed by Martin Co. }\end{array}$ & Special & J. Watcher & UNCL \\
\hline MND-P-2354 & $\begin{array}{l}\text { Nuclear 1.0-Watt Power } 6 / 60 \\
\text { Supply for Space Applications }\end{array}$ & Special & D. Knighton & UNCL \\
\hline
\end{tabular}

Three-Watt Polonium-210

Fueled Thermoelectric

Hazards Summary Report 6/59

Geled Thermoelectric

Hazards Summary Report $6 / 59$

for Two-Watt Promethium-147

ueled Thermoelectric Generator

Thermoelectric Power Supply

Process to be Performed by

Martin Company

Report of Americium Process

Supply for Space Applications 
II.

SNAP Development Programs - General Reports - CONTRACT AT(30-3)-217 (Cont'd)

\begin{tabular}{|c|c|c|c|c|}
\hline Report No. & Title & $\begin{array}{l}\text { Distri- } \\
\text { bution }\end{array}$ & Author & Classification \\
\hline MND-P-2355 & $\begin{array}{l}\text { Advance Thermoelectric } \\
\text { Power System Final Report }\end{array}$ & Limited & R. Harvey & UNCL \\
\hline MND-P-2356 & $\begin{array}{l}\text { Preliminary Operational } \\
\text { Safety Report for Thermo- } \\
\text { electric Generator }\end{array}$ & Special & D. Knighton & UNCL \\
\hline MND-P-2363 & $\begin{array}{l}\text { Preliminary Safety Analjsis } 6 / 60 \\
\text { Low Power Ce-144 Generator }\end{array}$ & Special & G. $D i x$ & UNCL \\
\hline MND-P-2364 & $\begin{array}{l}\text { Final Safety Analysis on } \\
\text { Polonium Fueled Generator }\end{array}$ & Special & C. Riggs & UNCL \\
\hline MND-P-2366 & $\begin{array}{l}\text { Preliminary Safety Analysis } 6 / 60 \\
\text { Report Curium Fueled Gen- } \\
\text { erator for Lunar and Satellite } \\
\text { Missions }\end{array}$ & Special & C. Riggs & UNCL \\
\hline MND-P-2372 & $\begin{array}{l}\text { Thermoelement Optimization } \\
\text { Code }\end{array}$ & Special & $\begin{array}{l}\text { T. Bustard } \\
\text { W. Lyon }\end{array}$ & UNCL \\
\hline MND-P-2373 & $\begin{array}{l}\text { 13-Watt Curium Fueled Thermo- } 7 / 60 \\
\text { electric Generator for Six- } \\
\text { Month Space Mission }\end{array}$ & Special & J. Bloom & UNCL \\
\hline MND-P-2374 & $\begin{array}{l}\text { Final Report on 13-Watt } \\
\text { Curium Fueled Thermoelectric } \\
\text { Generator for Hard Lunar } \\
\text { Impact Mission }\end{array}$ & Special & J. Bloom & UNCL \\
\hline $\begin{array}{l}\text { MND-FIIM-P- } \\
2144\end{array}$ & Nuclear Field Loading Mock-up 9/59 & Limited & - & SDI \\
\hline $\begin{array}{l}\text { MND-FILM-P- } \\
2146\end{array}$ & $\begin{array}{l}\text { Isotopic-Power Testing } \\
\text { for Space Use }\end{array}$ & Limited & - & UNCL \\
\hline
\end{tabular}


IIIA.

\begin{tabular}{|c|c|c|c|c|}
\hline Report No. & Title & $\begin{array}{l}\text { Distri- } \\
\text { bution }\end{array}$ & Author & sification \\
\hline MND-P-1229 & $\begin{array}{l}\text { Hazards Summary Report, } 1 / 58 \\
\text { SNAP I }\end{array}$ & Special & s. Clark & SRD \\
\hline MND-P-1519 & $\begin{array}{l}\text { SNAP I Re-Entry Eval- } 9 / 58 \\
\text { uation Study }\end{array}$ & Limited & G. Dix & SDI \\
\hline MND-P-1957 & $\begin{array}{l}\text { Test Program and Cell } \\
\text { Requirement for SNAP I }\end{array}$ & Limited & $\begin{array}{l}\text { W. Crane } \\
\text { D. Harvey }\end{array}$ & SDI \\
\hline MND-P-2128 & $\begin{array}{l}\text { SNAP I Dynamic Mercury } 4 / 60 \\
\text { Loop Tests of Selected } \\
\text { Material }\end{array}$ & Special & J. McGrew & UNCL \\
\hline MND-P-2309 & $\begin{array}{l}\text { Mercury Boiler Develop- } 6 / 60 \\
\text { ment Report SNAP I }\end{array}$ & Special & $\begin{array}{l}\text { J. Jicha } \\
\text { J. Keenan }\end{array}$ & UNCL \\
\hline MND-P-2350 & $\begin{array}{l}\text { SNAP I Radioisotope Fueled } \\
\text { Turboelectric Power } 6 / 60 \\
\text { Conversion System Summary }\end{array}$ & Special & P. Dick & UNCL \\
\hline MND-P-2375 & $\begin{array}{l}\text { SNAP I Power Conversion- } 6 / 60 \\
\text { System Development }\end{array}$ & Special & $\begin{array}{l}\text { Thompson-Ramo } \\
\text { Wooldridge }\end{array}$ & UNCL \\
\hline MND-P-2376 & $\begin{array}{l}\text { SNAP I Power Conversion- } 6 / 60 \\
\text { Turbine Development }\end{array}$ & $"$ & $"$ & $"$ \\
\hline MND-P-2377 & $\begin{array}{l}\text { SNAP I Power Conversion- } 6 / 60 \\
\text { Alternator Development }\end{array}$ & $"$ & $"$ & $n$ \\
\hline MND-P-2378 & $\begin{array}{l}\text { SNAP I Power Conversion- } 6 / 60 \\
\text { Pump Development }\end{array}$ & $"$ & $"$ & $"$ \\
\hline MND-P-2379 & $\begin{array}{l}\text { SNAP I Power Conversion- } 6 / 60 \\
\text { Bearing Derelopment }\end{array}$ & $"$ & $n$ & $"$ \\
\hline MND-P-2380 & $\begin{array}{l}\text { SNAP I Power Conversion- } 6 / 60 \\
\text { Controls Development }\end{array}$ & $"$ & $"$ & $"$ \\
\hline
\end{tabular}


IIIA.

SNAP-I CONTRACT AT(30-3)-217 TASK I -(Cont.)

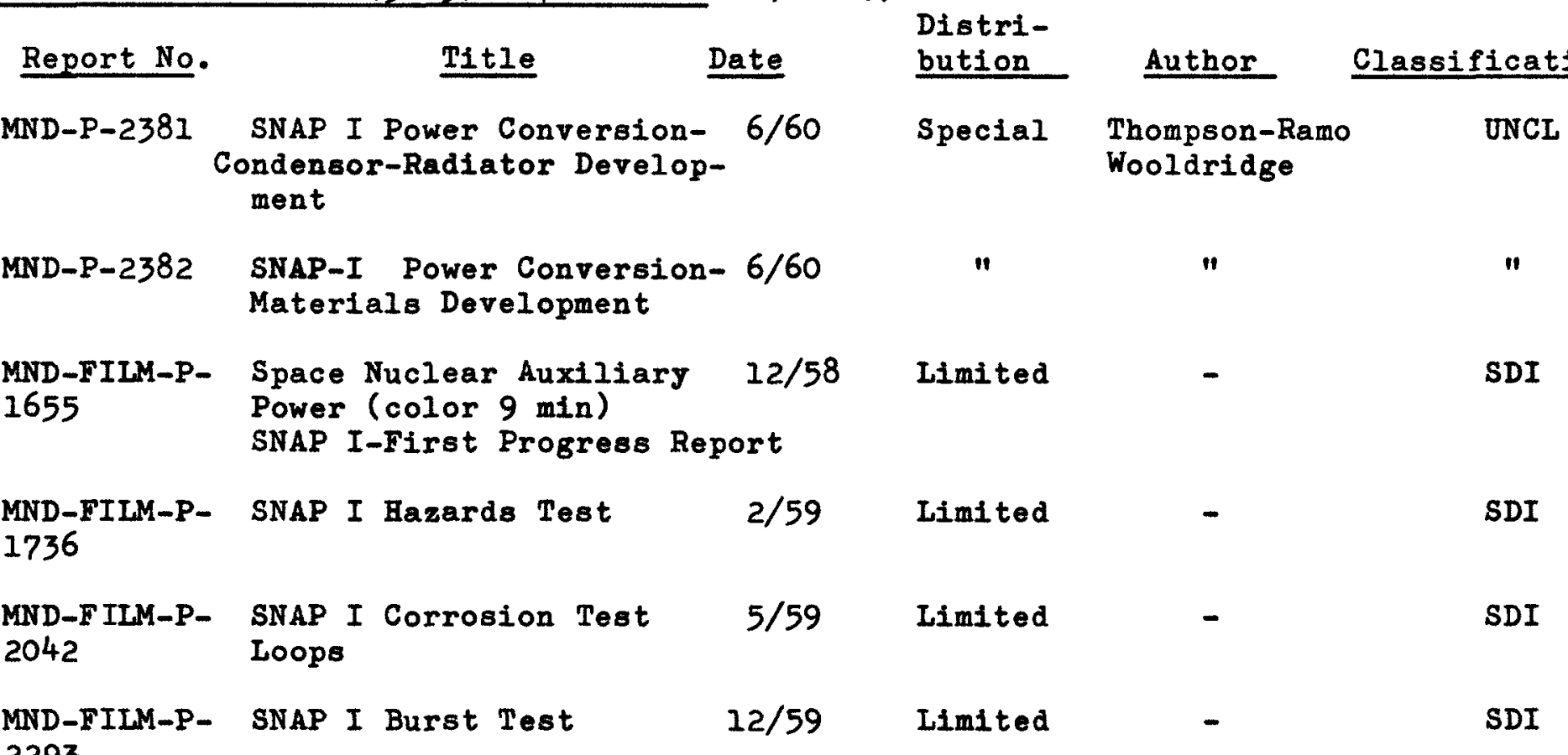




\begin{tabular}{|c|c|c|c|c|}
\hline Report No. & Title & $\begin{array}{l}\text { Distri- } \\
\text { bution }\end{array}$ & Author & Classification \\
\hline MND-P-2042-1 & $\begin{array}{l}\text { Marriage of } \triangle P U \text { and } 6 / 59 \\
\text { Nose Cone }\end{array}$ & Inited & - & SDI \\
\hline MND-P-2042-2 & Field Loading of APU $6 / 59$ & $n$ & - & CDI \\
\hline MND-P-2042-3 & $\begin{array}{l}\text { Quick Change Field } \\
\text { Loading Isotope } \\
\text { Cask }\end{array}$ & $n$ & - & UNC \\
\hline MND-P-2042-4 & $\begin{array}{l}\text { Field Loading Arrange-6/59 } \\
\text { ment }\end{array}$ & $"$ & - & SDI \\
\hline MND-P-2042-5 & $\begin{array}{l}\text { Operating Character- } 6 / 59 \\
\text { istics }\end{array}$ & $n$ & - & SRD \\
\hline MND-P-2042-6 & Energy Characteristice $6 / 59$ & " & - & SRD \\
\hline MND-P-2042-7 & $\begin{array}{l}\text { SNAP IA Thermoelec- } \\
\text { tric Generator }\end{array}$ & n & - & SDI \\
\hline MND-P-2042-8 & Transportation Dolly $\quad 6 / 59$ & $n$ & - & CDI \\
\hline & $\begin{array}{l}\text { NOTE: MND-P-2042-1 through } 81 \\
\text { slide and glossj print } 1 \\
\text { cards for each title and } \\
\text { on slide set. }\end{array}$ & $\begin{array}{l}\text { Includes } \\
\text { lecture } \\
\text { d commentary }\end{array}$ & & \\
\hline MND-P-2184 & $\begin{array}{l}\text { SNAP IA Preliminary } 2 / 60 \\
\text { Operational Hazards } \\
\text { Summary Report for Task } 2 \\
\text { Thermoelectric Generator }\end{array}$ & Special & G. Dix & SDI \\
\hline MND-P-2291 & $\begin{array}{l}\text { Summary Report, Aerodjnamic } \\
\text { Re-entry Analjsis, Task } 2 \text { 2/60 } \\
\text { Thermoelectric Generator }\end{array}$ & Special & R. Oehrli & SDI \\
\hline MND-P-2335 & $\begin{array}{l}\text { Interia Report on Safets } 6 / 60 \\
\text { Procedures for Task } 2 \\
\text { Thermoelectric Generator }\end{array}$ & Special & L. Klein & UNC \\
\hline MND-P-2352 & $\begin{array}{l}\text { Final Report on SNAP IA } 6 / 60 \\
\text { Hazards }\end{array}$ & Special & G. Dix & UNC \\
\hline
\end{tabular}




\begin{tabular}{|c|c|c|c|c|c|}
\hline Report No. & Title & ite & $\begin{array}{l}\text { Distri- } \\
\text { bution }\end{array}$ & Author & Classification \\
\hline $\begin{array}{l}\text { MND-P-2101-I } \\
\text { Vol. } 1\end{array}$ & $\begin{array}{l}\text { SNAP III Thermoelec- } \\
\text { tric Environmental } \\
\text { Tests }\end{array}$ & $8 / 59$ & Special & L. Gross & UNC \\
\hline $\begin{array}{l}\text { MND-P-2101-II } \\
\text { Vol. } 2\end{array}$ & $\begin{array}{l}\text { SNAP III Thermoelec- } \\
\text { tric Environmental } \\
\text { Tests }\end{array}$ & $10 / 59$ & Limited & L. Gros8 & SDI \\
\hline $\begin{array}{l}\text { MND-P-2101-III } \\
\text { Vol. } 3\end{array}$ & $\begin{array}{l}\text { SNAP III Thermoelec- } \\
\text { tric Environmental } \\
\text { Tests }\end{array}$ & $1 / 60$ & Special & $\begin{array}{l}\text { I. Gross } \\
\text { E. Schramm }\end{array}$ & ONC \\
\hline MND-P-2322 & $\begin{array}{l}\text { SNAP III Topical } \\
\text { Report }\end{array}$ & $2 / 60$ & Special & R. Harvej & UNC \\
\hline MND-P-2358 & $\begin{array}{l}\text { Nuclear Safety Test } \\
\text { Report for the } \\
\text { SNAP III Generator }\end{array}$ & $6 / 60$ & Special & T. Dobry & UNC \\
\hline MND-P-2368 & $\begin{array}{l}\text { Operational Testing } \\
\text { of SNAP III Generator }\end{array}$ & $6 / 60$ & Special & R. Wilson & UNC \\
\hline MND-P-2369 & $\begin{array}{l}\text { Conceptual Design of } \\
\text { a SNAP III Generator } \\
\text { Fueled with Ce-144 }\end{array}$ & $6 / 60$ & Special & R. Wilson & UNC \\
\hline MND-P-2370 & $\begin{array}{l}\text { Conceptual Design of } \\
\text { a SNAP III Type } \\
\text { Generator Fueled with } \\
\text { Po-2lo }\end{array}$ & $6 / 60$ & Special & R. Wilson & UNC \\
\hline
\end{tabular}


IIID.

SNAP-9A CONTRACT AT(30-1)-2871

\begin{tabular}{|c|c|c|c|c|c|}
\hline Report No. & Title & Date & $\begin{array}{l}\text { Distri- } \\
\text { bution }\end{array}$ & \multicolumn{2}{|c|}{ Classification } \\
\hline MND-P-2700-1 & $\begin{array}{l}\text { SNAP 9A Radiolsotope } \\
\text { Fueled Thermoelectric } \\
\text { Power Conversion System } \\
\text { Development. } \\
\text { Quarterly Progress } \\
\text { Report No. } 1\end{array}$ & $11 / 61$ & Special & Paul J. Dick & CRD \\
\hline MND-P-2700-2 & $\begin{array}{l}\text { Quarterly Progress } \\
\text { Report No. } 2\end{array}$ & $2 / 62$ & $"$ & Paul J. Dick & CRD \\
\hline MND-P-2700-3 & $\begin{array}{l}\text { Quarterly Progress } \\
\text { Report No. } 3\end{array}$ & $5 / 62$ & $"$ & Charles R. Fink & CRD \\
\hline MND-P-2725 & $\begin{array}{l}\text { Specification for Therma } \\
\text { Environment Test SNAP } \\
\text { 9A-SNAP II }\end{array}$ & $2 / 62$ & $"$ & T. J. Dobry & CRD \\
\hline MND-P-2775 & $\begin{array}{l}\text { Preliminary Safety } \\
\text { Analysis SNAP 9A } \\
\text { Transit Mission }\end{array}$ & $4 / 62$ & $"$ & T. J. Young & CRD \\
\hline MND-P-2775-2 & $\begin{array}{l}\text { SNAP 9A Radioisotope- } \\
\text { Fueled Generator Final } \\
\text { Safety Analysis for } \\
\text { Transit Mission }\end{array}$ & $3 / 63$ & $C-92 A$ & T. J. Dobry & SRD \\
\hline MND-P-2809 & $\begin{array}{l}\text { Instructional Manual - } \\
\text { SNAP 9A Electric Gen- } \\
\text { eration System }\end{array}$ & $3 / 63$ & C-92A & MND & CRD \\
\hline MND-P-2874 & $\begin{array}{l}\text { Feasibility Report No. } \\
8 \text { for Transfer and Test } \\
\text { of SNAP 9A Units at John } \\
\text { Hopkins Applied Physics } \\
\text { Laboratory }\end{array}$ & $2 / 63$ & C-92A & MND & UNC \\
\hline
\end{tabular}




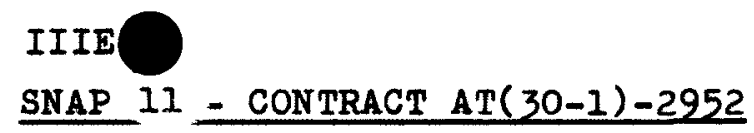

SNAP 12 - CONTRACT AT $(30-1)-2952$

\begin{tabular}{|c|c|c|c|c|c|c|}
\hline \multirow{2}{*}{$\begin{array}{l}\text { Report No. } \\
\text { MND-P-2811-1 }\end{array}$} & \multicolumn{2}{|r|}{ Title } & \multirow{2}{*}{$\begin{array}{l}\text { Date } \\
4 / 62\end{array}$} & \multirow{2}{*}{$\begin{array}{l}\text { Distri- } \\
\text { bution } \\
\text { C-92A }\end{array}$} & \multirow{2}{*}{$\begin{array}{l}\text { Author } \\
\text { MND }\end{array}$} & \multirow{2}{*}{$\begin{array}{l}\text { Classification } \\
\text { CDI }\end{array}$} \\
\hline & $\begin{array}{l}\text { SNAF } \\
\text { Prog } \\
\text { Quar }\end{array}$ & $\begin{array}{l}\text { ll-Surveyor } \\
\text { ram } \\
\text { terly Progress }\end{array}$ & & & & \\
\hline MND-P-2811-2 & 1 & $" 1$ & " 7/62 & 11 & $n$ & $"$ \\
\hline MND-P-2811-3 & n & $"$ & " $10 / 62$ & " & $"$ & $"$ \\
\hline MND-P-2811-4 & " & $" 1$ & $11 / 63$ & $"$ & $n$ & $"$ \\
\hline
\end{tabular}


IIIF.

IMP - CONTRACT AT $(30-1)-3169$

REPORT NO.

MND-P-2989-1

\section{TITLE}

Radiolsotope Power Supply 5/63

For The Interplanetary

Monitoring Probe Satellite

Program. Quarterly Progress Report No. 1
DATE DISTRIBUTION AUTHOR CLASSIFICATION

C-92A

MND

C.R.D. 


\begin{tabular}{|c|c|c|c|c|c|}
\hline Report No. & Title & Date & Distri- & Author & sifica \\
\hline MND-P-2483-2 & $\begin{array}{l}\text { SNAP-7 Programs - Strontium } \\
90 \text { Fueled Thermoelectric } \\
\text { Generator Development } \\
\text { Quarterly Progress Report }\end{array}$ & $4 / 61$ & Special & W. West & UNCL \\
\hline MND-P-2483-3 & $"$ & $7 / 61$ & Speclal & W. West & $"$ \\
\hline MND-P-2483-4 & $"$ & $10 / 61$ & Speclal & W. West & $"$ \\
\hline MDD-P-2483-5 & $"$ & $1 / 62$ & Special & W. A. McDonald & $"$ \\
\hline MND-P-2483-6 & $"$ & $4 / 62$ & Special & W. A. McDonald & $"$ \\
\hline MND-P-2483-7 & $"$ & $7 / 62$ & Speclal & W. A. McDonald & $"$ \\
\hline MND-P-2613 & $\begin{array}{l}\text { SNAP-7A Final Safety Analy- } \\
\text { sis-Ten Watt Strontium-90 } \\
\text { Fueled Generator for an Un- } \\
\text { attended Light Buoy }\end{array}$ & $1 / 62$ & Special & MND & " \\
\hline MND-P-2720 & $\begin{array}{l}\text { SNAP-7A Strontium-90 Fuelea } \\
\text { Thermoelectric Generator Power } \\
\text { Source for Five-Watt U. S. } \\
\text { Coast Guard Light Buoy Final } \\
\text { Report }\end{array}$ & $\mathrm{r}^{2 / 62}$ & Special & MND & $"$ \\
\hline MND-P-2661 & $\begin{array}{l}\text { Instructional Manual-SNAP-7A } \\
\text { Electric Generation Station }\end{array}$ & $1 / 62$ & Special & MND & $"$ \\
\hline MND-P-2614 & $\begin{array}{l}\text { SNAP-7C Final Analysis-Ten } \\
\text { Watt Strontium-90 Fueled } \\
\text { Generator for an Unattended } \\
\text { Meterological Station }\end{array}$ & $5 / 61$ & Special & MND & $"$ \\
\hline MND-P-2707 & $\begin{array}{l}\text { SNAP-7C Strontium-90 Fueled } \\
\text { Thermoelectric Generator } \\
\text { Power Source Five-Watt U.S. } \\
\text { Navy Weather Station - Final } \\
\text { Report }\end{array}$ & $8 / 61$ & Special & MND & $"$ \\
\hline
\end{tabular}


Report No.

MND-P-2640

MND-P-2762

ND-P-2834

MND-P-2664A

MND-P-2835

MND-P-2786

MND-P-2836

\section{Title}

Instruction Manual SNAP-7C Electric Generation System

SNAP-7B Final Safety Evalua- 4/62 tion of a Sixty Watt Strontium -90 Fueled Generator for a

U. S. Coast Guard Automatic Light Station

SNAP-7B Strontium-90 Fueled

Power Source - Thirty Wate

U. S. Coast Guard Automatic Light Station

Instruction Manual - SNAP-7B Electric Gneeration System

SNAP-7D Final Safety Evalua- 5/62

tion of a Sixty Watt Strontium

-90 Fueled Generator for a

U. S. Navy Boat Type Weather

Station

SNAP-7D Strontium-90 Fueled 3/63

Thermoelectric Generator Power

Source. Thirty-Watt U. S.

Navy Floating Weather Station

Instruction Manual Snap-7D 3/63

Electric Generation System
Bustri-

Author

Classification

Special MND

UNCL

Special V. G. Kelly

H. N. Berkow

11

Special

c. N. Young

II

Special MND

"

Special V. G. Kelly

H. N. Berkow

Special

C. N. Young

"

Specia1 MND 
IVB.

SNAP 7E - CONTRACT AT (30-1)-2958

REPORT NO.

TITLE

DATE DISTRIBUTION AUTHOR CLASSIFICATION

MND-2821

Instruction Manual SNAP-

$6 / 62$

Special

MND

UNCL

System

MND-P-2761

Final Safety Evaluation

$5 / 62$

Spec1al

H.N. Berkow, of a Ten Watt Strontium90 Fueled Generator For

V.G. Kelly

A Deep Sea Application

MND-P-2837

SNAP 7E Sr-90 - Fueled

Thermoelectric Generator

$7 / 62$

for an Undersea Beacon -

Final Report 
IVC. CESIUM-132 GENERATOR

Report No. Title

RRC-C8-0100

Cs-137 Fueled Generator
Distri-

bution Author Classification

Date bution

$?$

Royal $?$

$?$

Research Corp.

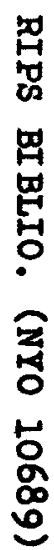




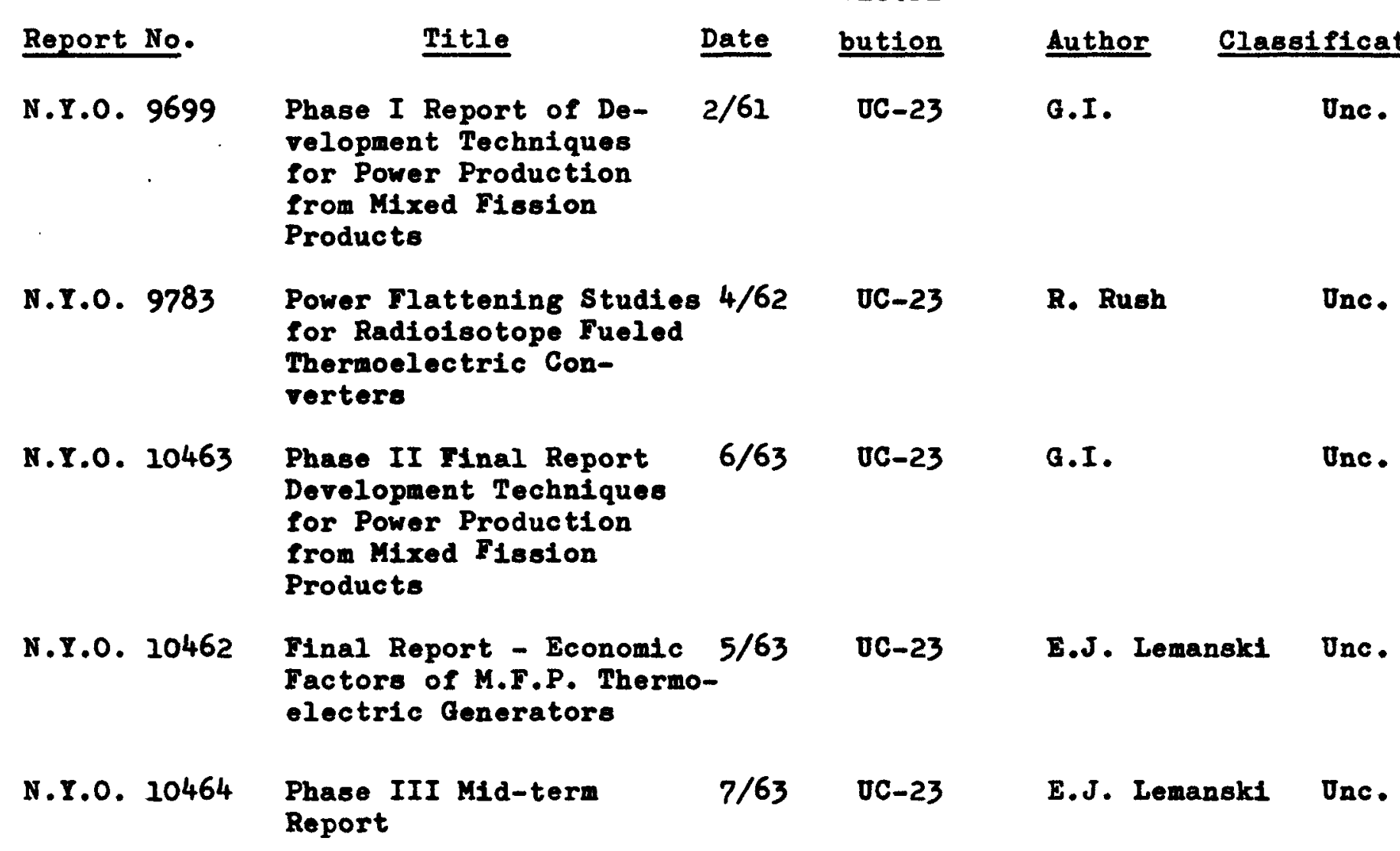


VA.

Advancod SNAP TechnologY - CONTRACT AT(30-3)-217 TASK V and SNAP-13 Contract AT $(30-1)-3060$

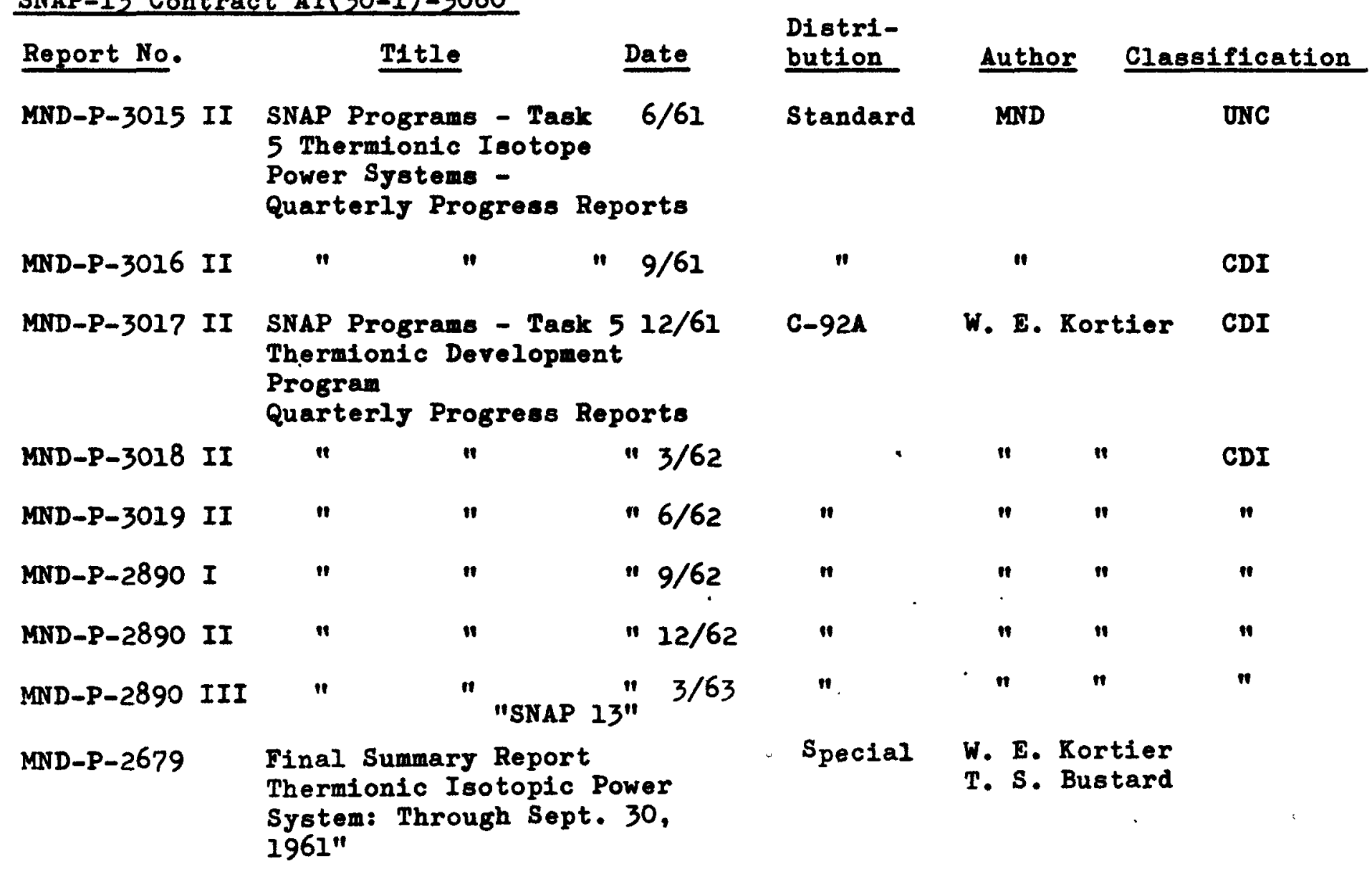


VA.

Barium Vapor Filled Thermionic Plasma Energy Converters - CONTRACT AT(30-1)-2933

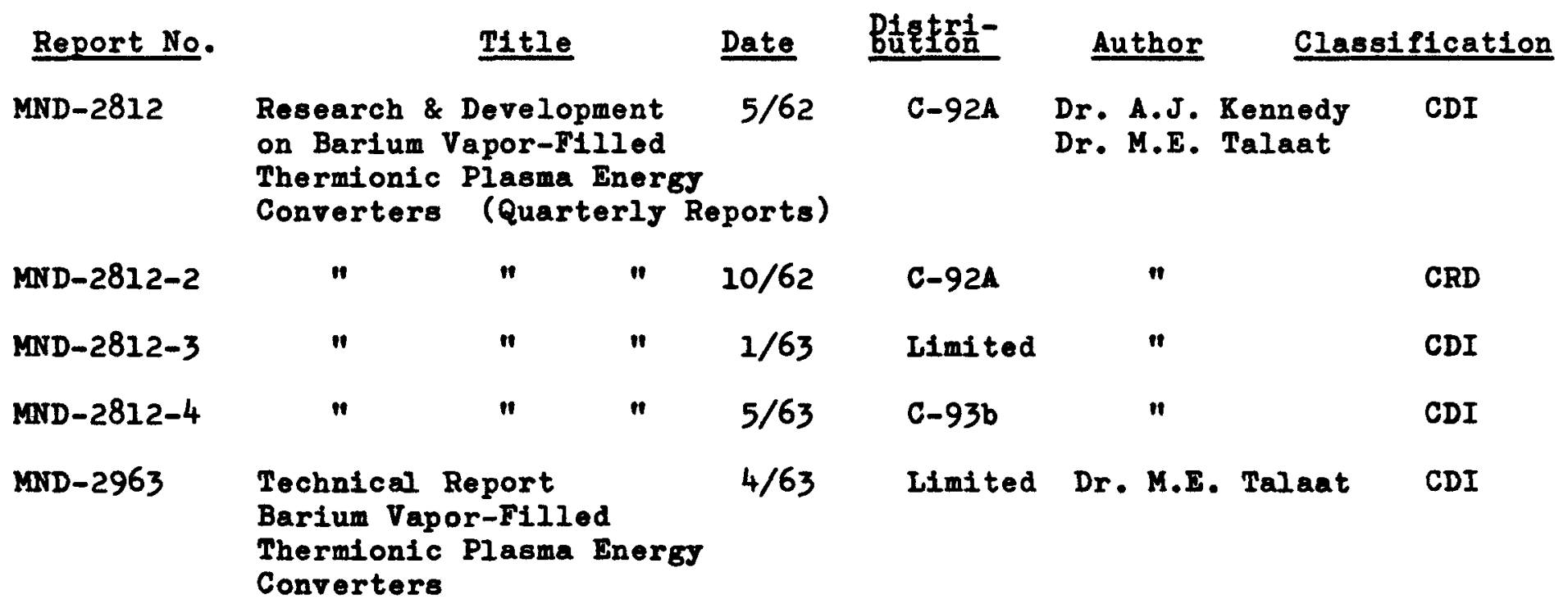


VB. OTHER RESEARCH AND DEVELOPMENT REPORTS

\begin{tabular}{|c|c|c|c|c|c|c|}
\hline Report No. & Pitle & Date & $\begin{array}{l}\text { Distri- } \\
\text { bution }\end{array}$ & Author & \multicolumn{2}{|c|}{ Classification } \\
\hline MND-P-2801 & $\begin{array}{l}\text { SNAP Programs Final Summary } \\
\text { Report Task } 6 \text { Fuel Tech- } \\
\text { nology Development Program }\end{array}$ & $7 / 62$ & C-92A & MND & & CRD \\
\hline MND-P-2953 & $\begin{array}{l}\text { SNAP Programs Upper Atmos- } \\
\text { phere Experimental Re-entry } \\
\text { Study - Final Summary Report }\end{array}$ & $4 / 63$ & C-92A & William & Hagis & CRD \\
\hline $\begin{array}{l}\text { H.W. } 71319 \\
\text { Rev. }\end{array}$ & $\begin{array}{l}\text { Special Radioisotopes for } \\
\text { Power }\end{array}$ & $10 / 16 / 61$ & $?$ & C. A. R & ohrmann & $?$ \\
\hline H.W. 76323 & Radioisotopic Heat Sources & $2 / 1 / 63$ & vC-2 & C. A. R & ohrmann & UNCL \\
\hline
\end{tabular}


APPENDIX I

SELBCTED ADDITIONAL REFERENCES IN THE UNCLASSIFIED LITERATURE

1. Space Nuclear Power Applications - Hearings before the Subcommittee on Research, Development, and Radiation of the Joint Committee on Atomic Energy, Congress of the U. $S$.

U. S. Government Printing Office, Washington, Sept. 1962

2. Radionuclide Power for Space:

a. Davis, H. - "Part I: Isotope Cost and Availability," Nucleonics 21-3, 61-65, Mar. 163

b. Harvey, D. G., P. J. Dick and C. R. Fink, "Part II Isotope Generator Reliablitty and Safety", Nucleonics, $21-4,56-59$, Apr. 63

3. Nuclear Energr in Space - Nucleonics, 19-4, 54-100; Apr. 61 Comprehensive review of Nuclear space programs including:

a. Harvey, D. and J. G. Morse - "Electric Power Sources": Radionuclide Power for Space Mission", pages 69-72

b. Branch, I. I. and J. A. Connor, Jx. - "Nuclear Safety in space", pages 64-68

4. American Rocket Society, Progress in Astronautics and Rocketry series

Snyder, N. W., Ed., Vol 4: SPACE POWER SYSTEMS, 1961, 632 pages Sertes of Technical Papers on Solar, Nuclear, and Chemical systems and Power Requirements, Including:

a. Bloom, J. I. and Wedell, J. B., "Thirteen Watt Isotope Powered Thermoelectric Generators for space and Lunar Impact Missions," pp 485-517

b. Greenfleld, H. H., "Optimized SNAP III Power Generator Design for Spacecraft," pp 519-546

c. Dick, P. J., "Safety Analyses and Tests of a Radioisotope Powered Thermoelectric Generator," pp 547-561

5. Other Published Journal Articles

a. Morse, J. G., "Energy for Remote Areas," Science, 139-3560, 1175-1180, Mar. 22, 1963

b. Corliss, W. R., "Power Sources for Nuclear Space Instruments," Nucleonics, 20-10, 61-63, Nov. 62 


$$
\text { APPENDIX I - } 2
$$

c. Kershaw, W. L., "Radioisotope Fueled Thermoelectric Generators," Electro Technoloxy, July 1962

d. Harvey, D. G., "Integrating Isotopic Power Systems," Astronautics, May 1962

e. Morse, J. G., and Harvey, D. G., "Nuclear Energy in Space Radioisotope Auxillary Power Systems, "Aerospace Engineering, November 1961

f. Crompton, C. E., "Isotopic Power,", Industrial Research, October 1961

8. Morse, J. G., "Isotopic Power, "The Militery Engineer, January-February, 1961

h. Huffman, F. N., and Gross, I. W., "Performance Data and Environment Test Results of SNAP III," Ballistic Missiles and Space Technologr, Vol. II, 1961

1. Hagis, W., Dobry, T. and Dix, G., "Nuclear Safety of SNAP III for Space Missions," ARS Journal, Dec. 6I

6. Forecasts of Bpace Isotopic Power Requirements

a. "NASA Increases Estimate of Isotopic Power Needs," Forum

Memo, Atomic Industrial Forum, Inc.. NY, June 1963

b. "Space Applications of Nuclear - Electric Power", Radio

Corporation of America N. Y., AED-P5013, March 1963

c. "Nucleonics in Space," Nucleonics Markets, Vol. 5, No. 2, Oct/Dec. 62 (McGraw-Hill) 
APPENDIX II Summary of Radioisotove Power Supplies

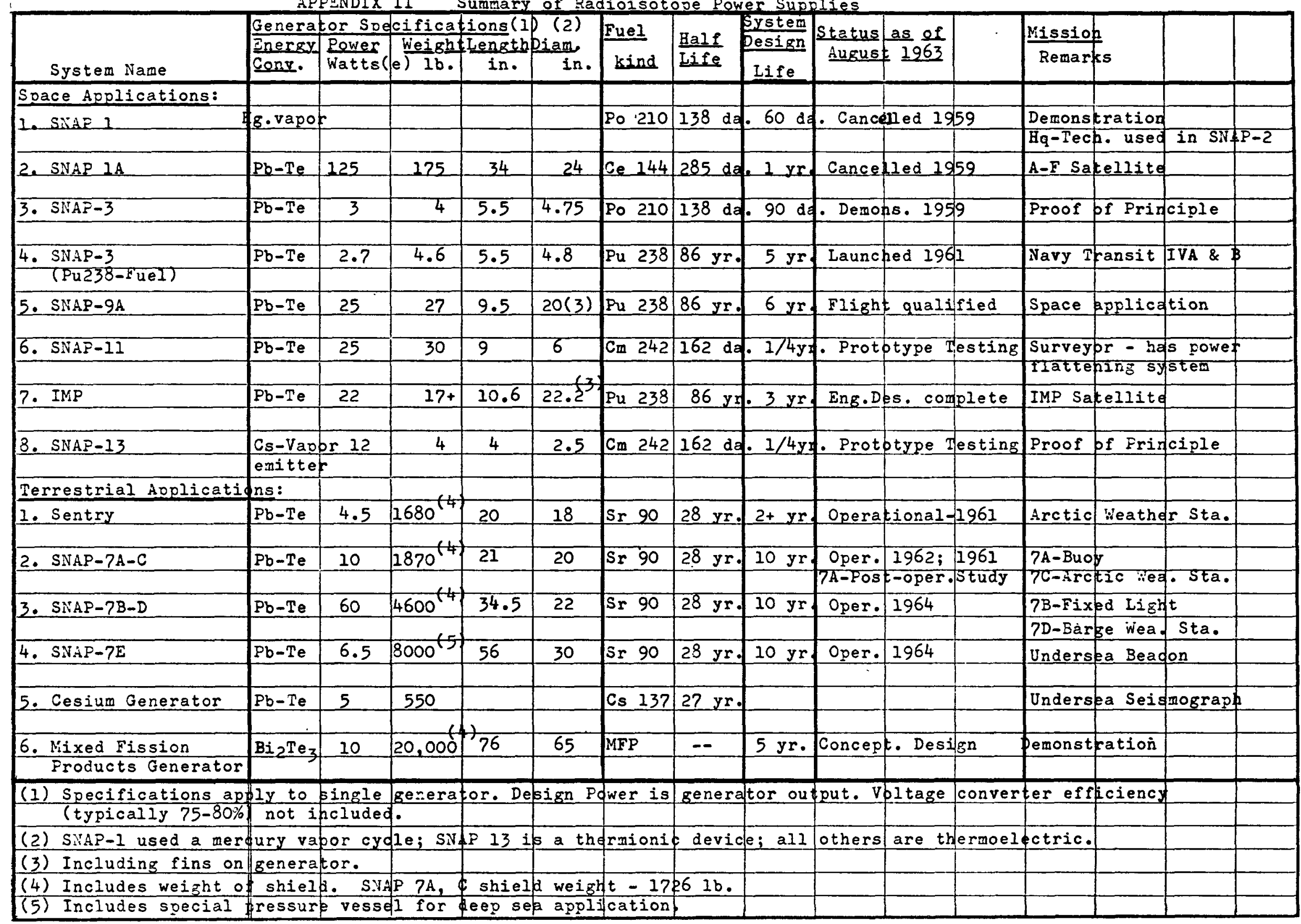


APPENDIX III - SAMPLE LIST OF SPECIFICATIONS AND DRAWINGS FOR A RADIOISOTOPE POWER SUPPLI

(RIPS FOR INTERPLANETARY MONITORING PROBE)

\begin{tabular}{|c|c|}
\hline \multicolumn{2}{|r|}{ SPECIFICATIONS } \\
\hline $\begin{aligned} \text { MN-10073, } & \text { Rev } 1 \\
\text { June } 6, & 1963\end{aligned}$ & $\begin{array}{l}\text { "Specification for a Radiolsotope Fueled Power } \\
\text { Supply for Interplanetary Monitoring Probe } \\
\text { Satellites" } \\
\text { (C-DI) }\end{array}$ \\
\hline $\begin{aligned} \text { MN-10074, } & \text { Rev. } 1 \\
\text { June } 7, & 1963\end{aligned}$ & $\begin{array}{l}\text { "Specification for Environmental Conditions and } \\
\text { Environmental Tests for a Radioisotope-Fueled } \\
\text { Power Supply for Interplanetary Monitoring Probe } \\
\text { Satellites" }\end{array}$ \\
\hline $\begin{array}{c}\text { MN-10075, Rev. } 1 \\
\text { June 10, } 1963\end{array}$ & $\begin{array}{l}\text { "Specification for an Electric Converter/Regulator } \\
\text { for a Radioisotope-Fueled Power Supply for the } \\
\text { IMP-C Satellite" }\end{array}$ \\
\hline $\begin{array}{l}\text { MN-10076, Rev. } 1 \\
\text { June 11, } 1963\end{array}$ & $\begin{array}{l}\text { "Specification for a Safety Program for a Radio- } \\
\text { isotope-Fueled Generator for Interplanetary } \\
\text { Monitoring Probe Satellites" }\end{array}$ \\
\hline $\begin{array}{l}\text { To be developed } \\
\text { To be developed }\end{array}$ & $\begin{array}{l}\text { Specification for Fuel Capsules for A Radio- } \\
\text { isotope-Fueled Power Supply for Interplanetary } \\
\text { Monitoring Probe Satellites } \\
\text { Quality Control Procedures } \\
\text { DRAWINGS }\end{array}$ \\
\hline
\end{tabular}

Master Assembly Drawings

439 A 1110000

439 A 1110001

To be released
Generator Assembly Drawing (C-RD)

Generator External installation

Generator Assembly Torquing Instructions

Heat Source Items

To be released NSK -89

439 A 1110151

439 A 1110153

439 A 1110157
Fuel capsule (C-RD)

Graphite Block Assembly (fueled units)

Mica Sheet

Capsule end support (fueled units)

Stainless Steel Disc Load Distributor (fueled units)

Energy Conversion Items

$\begin{array}{rrl}439 \text { A } 1110200 & \text { Module Assembly } \\ \text { " } & 0201 & \text { Couple Assembly } \\ \text { " } & 0250 & \text { Shoe, Hot Junction } \\ \text { " } & 0251 & \text { Thermoelectric Element } \\ \text { " } & 0252 & \text { Shoe, Cold Junction } \\ & 0253 & \text { Insulation, Module Strip }\end{array}$


DRAWINGS (Continued)

Energy Conversion Items

$\begin{array}{lll}439 \text { A } 111 & 0254 & \text { Lug, Terminal (2 sheets) } \\ \text { " } & 0255 & \text { Piston and Button (Alignment Details) } \\ \text { " } & 0256 & \text { Module Bar } \\ \text { " } & 0258 & \text { Insulation Header Blocks (fueled units) }\end{array}$

Heat Refection and Containment Items

$\begin{array}{ccc}439 \text { A } & 111 & 0300 \\ \text { " } & 0301 \\ \text { " } & 0350 \\ \text { " } & 0351 \\ \text { " } & 0353 \\ \text { " } & 0354 \\ & 0355\end{array}$

Housing Acsembly

Corer, Opper

Body, Housing

Fin

Bolt, Extension

Connector Holddown Ring and Shim

Cover, Bottom (fueled units)

PN $8100 \quad 000$

Hermetically Sealed Electrical Connector

To be released

Generator Finish Specification

Other Items

439 A 1110400

Wiring Diagram Schematic

Installation Tool ( 3 sheets)

To be released

$D C-D C$ Converter Drawings

Additional or Substitute Drawings for Prototype Units Only

PN 6400000

439 A 1110150

" 0152

" 0156

" 0257

" 0352
Heater Cartridge (Fire rod)

Adapter Electric Heater

Stainfess steel Disc Load Distributor

Disc, mica

Insulation Header Blocks

Cover, Bottom

Note: Additional drawings are required for such items as mockup, test fixtures, conceptual and alternate design studies. 